\title{
Treatment Considerations for Patients with Unresectable Metastatic Melanoma Who Develop Pembrolizumab-Induced Guillain-Barré Toxicity: A Case Report
}

\author{
Sivraj Muralikrishnan ${ }^{a} \quad$ Lara K. Ronan $^{\mathrm{b}}$ Shodeinde Coker ${ }^{\mathrm{c}}$ \\ Paula K. Rauschkolb ${ }^{d}$ Keisuke Shirai ${ }^{a}$ \\ aDepartment of Hematology/Oncology, Dartmouth-Hitchcock Medical Center, Lebanon, \\ $\mathrm{NH}, \mathrm{USA} ;{ }^{b}$ Department of Neurology and Medicine, Dartmouth-Hitchcock Medical Center, \\ Lebanon, NH, USA; 'Bristol-Myers Squibb Co., Lawrenceville, NJ, USA; dNorthern Arizona \\ Healthcare, Flagstaff, AZ, USA
}

\section{Keywords}

Immunotherapy · Guillain-Barré toxicity · Melanoma $\cdot$ Pembrolizumab · Ipilimumab

\begin{abstract}
Immunotherapy has improved outcomes in many malignancies, most notably in melanoma, lung cancer, and bladder cancer. Understanding the side effects associated with these medications is an important part of managing our patients. Although fatigue, rash, and diarrhea are commonly reported side effects, it is important to be cognizant of rarer ones, such as neuropathy. Amongst the different neurological toxicities that have been reported in the literature, Guillain-Barré-like neuropathies are quite rare. However, the occurrence of such neuropathies in a patient can be life threatening. The problem this poses in treating cancers such as melanoma is that it eliminates an effective class of medication available to the patient, which can ultimately affect their prognosis. We present a case of a 65 -year-old female with unresectable metastatic melanoma who developed Guillain-Barré-like neuropathy after two doses of pembrolizumab. Her clinical course was complicated by three separate hospitalizations over 3 months due to recurring bouts of neuropathy, which resulted in a significant decline in performance status and delay in subsequent treatment of her melanoma. Her prolonged recovery eventually resulted in progression of her melanoma nearly 1 year later, while off therapy. Instead of discontinuing immunotherapy completely, she agreed to a re-challenge with ipilimumab. After one dose, her melanoma regressed and continues to show a sustained response nearly 1 year after treatment without any signs of relapse in her neuropathy. Guillain-Barré toxicity resulting from immune checkpoint inhibition poses a difficult chal-
\end{abstract}


Muralikrishnan et al.: Treatment Considerations for Pembrolizumab-Induced GBS

Toxicity in Melanoma

lenge to an oncologist who is determining the next line of treatment for patients with unresectable metastatic melanoma that have progressed while off therapy and who have no targetable mutations. Our case raises the question of whether a re-challenge with a different class of immunotherapy agent is a reasonable option.

(c) 2020 The Author(s)

Published by S. Karger AG, Basel

\section{Introduction}

The adoption of immunotherapy in clinical practice has resulted in significant improvements in the outcomes of lung cancer, melanoma, and bladder cancer [1-3]. However, increasing use of these agents have also led to increasing incidence of significant and sometimes fatal autoimmune adverse effects. In a retrospective review of 3,545 patients treated with immune checkpoint inhibitors from 7 academic centers, Wang et al. [4] found a $0.6 \%$ fatality rate related to immune checkpoint inhibition, with $43 \%$ of these fatalities related to a cardiac or neurological event. Using the VigiLyze Database (WHO database of individual safety case reports and adverse drug reactions), Wang et al. also found that pneumonitis (35\%), hepatitis (22\%), colitis (17\%), and neurological events (15\%) were the most common causes of fatal toxicity with anti-PD-1/PD-L1 therapy while colitis (70\%), hepatitis $(16 \%)$, and pneumonitis ( $8 \%)$ were the most common causes of fatality with ipilimumab.

The early recognition, diagnosis and management of autoimmune side effects is critical for achieving optimal outcomes in our patients. The National Comprehensive Cancer Network (NCCN) published a set of clinical guidelines to assist clinicians in their management of these side effects. Compared with commonly reported adverse effects such as fatigue and rash, whose incidences are estimated to be $16-24 \%$ and $34-39 \%$, respectively [5], neurological toxicity is less common. While encephalitis and myasthenia gravis make up the majority of fatal neurological toxicities from immune checkpoint inhibition [4], Guillain-Barré-like syndromes (GBS) have also been reported with the use of ipilimumab, nivolumab, and pembrolizumab [6-15]. However, the clinical outcomes for some of these cases varies. For instance, in the case reported by Wilgenhof and Neyns [6], a 57-year-old female with recurrent melanoma developed a rapidly ascending loss of sensory and motor function of the limbs after three doses of ipilimumab. She was diagnosed with GBS and treated using steroids with a taper over 18 weeks. After permanently discontinuing ipilimumab, her positron emission tomography (PET) scans at 12 and 24 weeks had shown regression of her disease. Conversely, Manam et al. [15] describe a case of an 81-year-old male with metastatic melanoma to the brain who developed progressive lower and upper extremity weakness and areflexia 1 month after his second cycle of pembrolizumab. His workup was suggestive of an acute inflammatory demyelinating polyneuropathy that prompted the initiation of intravenous steroids and intravenous immunoglobulin (IVIG). His course was complicated by hypoxic respiratory failure requiring mechanical ventilation. Since he did not respond to IVIG, plasma exchange was initiated. Unfortunately, on day 14 of hospitalization, he developed seizures that were found to be secondary to hemorrhage within one of his metastatic brain lesions. Care was eventually withdrawn, and the patient died shortly thereafter. Both of these cases highlight the difficulty that exists in predicting the outcome for patients who develop Guillain-Barrélike neuropathies as a result of immunotherapy. According to NCCN guidelines, for any grade of GBS, the recommendation is to permanently discontinue immunotherapy. In patients with metastatic unresectable melanoma without targetable mutations, this presents a difficult challenge for the clinician in terms of determining the next line of treatment. 
Muralikrishnan et al.: Treatment Considerations for Pembrolizumab-Induced GBS

Toxicity in Melanoma

Here, we describe a case of re-challenging a patient with a different class of immunotherapy for progression of her unresectable metastatic melanoma, despite developing a grade 3 Guillain-Barré-like neuropathy after two doses of pembrolizumab.

\section{Case Presentation}

The patient is a 65-year-old female with no significant past medical history who presented to the Oncology Department with a newly diagnosed malignant melanoma first noticed on a screening mammogram. She underwent core needle biopsy of a right-sided breast nodule that confirmed the diagnosis. Staging PET scan revealed several FDG-avid subcutaneous nodules in the chest, right flank, and right upper arm and an excisional biopsy of one of these lesions confirmed metastatic disease. Her melanoma was BRAF/CKIT/NRAS negative. She started treatment with pembrolizumab $200 \mathrm{mg}$ every 3 weeks.

Within a few hours of completing her second cycle of pembrolizumab, she reported an ascending numbness in her feet and ankles bilaterally that radiated up to her knees. The patient subsequently underwent brain magnetic resonance imaging that was negative. Unfortunately, due to worsening symptoms, which now included lower extremity muscle weakness with gait instability, left hand weakness, and left tongue numbness, she had a neurological evaluation that raised suspicion of an immunotherapy-associated neuropathy. She presented back to our oncology clinic where it was determined that she required admission for worsening neurological symptoms. Her physical exam on admission was notable for $4 / 5$ strength of the left upper extremity with weaknesses in arm abduction at the shoulder, elbow extension/ flexion, hand grip and finger flexion. She also had 4/5 strength with left foot plantar flexion and dorsiflexion. Reflexes were diminished at the biceps and brachioradialis bilaterally and nonexistent in the patellar and Achilles tendon bilaterally. Her tandem gait was unsteady, but Romberg was negative. She also had diminished facial sensation along the left V1 distribution.

During her hospitalization, her cerebrospinal fluid (CSF) analysis showed clear appearance with a normal glucose $(63 \mathrm{mg} / \mathrm{dL}), 17 / \mathrm{mm}^{3}$ nucleated cells, $42 / \mathrm{mm}^{3}$ red blood cells with an elevated protein level (total protein $78 \mathrm{mg} / \mathrm{dL}$ [normal range: 15-45]), albumin $43.3 \mathrm{mg} / \mathrm{dL}$ (normal range: <27), with the presence of oligoclonal bands in CSF. Testing for anti-ganglioside and anti-myelin-associated glycoprotein was negative. A nerve conduction study during this admission was not suggestive of neuropathy either. However, given the clinical suspicion for GBS, she received treatment with 5 days of IVIG, leading to a significant improvement in her muscle weakness. Unfortunately, 18 days after discharge, she developed an ascending paralysis of her upper and lower extremities that was complicated by a fall, which resulted in an ankle fracture and a newly diagnosed pulmonary embolism. On this admission, she received plasma exchange for 7 days and intravenous solumedrol for 5 days, instead of IVIG. This time, the plan was to discharge her on a slow taper of oral prednisone. After seeing some improvement in her symptoms, she was discharged to an acute rehabilitation facility and eventually went home. However, 44 days after her second admission, she was re-admitted for worsening ascending paralysis complicated by spasticity in her toes and fingers. A repeat nerve conduction study on this admission showed slowing of waveforms that was suggestive of an acute and chronic demyelinating polyneuropathy. A repeat lumbar puncture was performed with CSF analysis showing clear appearance with a normal glucose (62 $\mathrm{mg} / \mathrm{dL}$ ), 3/ $\mathrm{mm}^{3}$ nucleated cells, $0 / \mathrm{mm}^{3}$ red blood cells with an elevated protein level (total protein $175 \mathrm{mg} / \mathrm{dL}$ [normal range: 15-45]), albumin $96.9 \mathrm{mg} / \mathrm{dL}$ (normal range: <27), and IgG $10.8 \mathrm{mg} / \mathrm{dL}$ (normal range: $<8.1$ ) but no oligoclonal bands. Testing for leukemia and lymphoma in the CSF was negative. She once again received 7 days of plasma exchange and 5 days of intravenous solumedrol but was discharged on a higher dose of oral prednisone than 
Muralikrishnan et al.: Treatment Considerations for Pembrolizumab-Induced GBS

Toxicity in Melanoma

before and a planned taper over the course of many weeks. In addition to this, she received periodic plasma exchanges as an outpatient.

Due to the development of Guillain-Barré-like neuropathy, pembrolizumab was stopped and her melanoma was followed with PET scans every 2 months. After initially showing a mixed response, progression occurred about 1 year after her last dose of pembrolizumab. This included several new subcutaneous nodules and a new brain lesion for which she underwent stereotactic radiosurgery. After a long discussion about the risks and benefits of re-challenging with a different class of immunotherapy, the patient agreed to start with ipilimumab, a CTLA-4 inhibitor. She only received 1 dose of ipilimumab with PET scans showing a significant and sustained response nearly 1 year later. Her only reported symptoms after receiving ipilimumab were diarrhea and ongoing bilateral leg tingling. She remains on a low dose of steroids at this time but has not had any re-occurrence of her Guillain-Barré-like neuropathy.

\section{Discussion}

The impact of immunotherapy in melanoma has improved outcomes in a disease that has been historically difficult to treat. The effects of immunotherapy can be longstanding even after treatment discontinuation as suggested by the 4-year survival and outcome results of the KEYNOTE-006 study, presented by Long et al. [16] at the 2018 American Society of Clinical Oncology (ASCO) Annual Meeting. In this abstract, it was found that $86 \%$ of patients who completed 2 years of pembrolizumab treatment for unresectable stage III-IV melanoma were alive at the 4-year mark. Similarly, in the 4-year follow-up of the CheckMate 067 study published by Hodi et al. [17], 71\% of patients with advanced melanoma receiving combination therapy with ipilimumab and nivolumab were treatment free, further supporting the idea of a sustained response even after stopping immunotherapy. In patients with unresectable metastatic melanoma, immunotherapy is typically considered the first-line therapy unless a targetable mutation is present and a quick response is desired. In those without a targetable mutation, immunotherapy is typically the best option available and offers the best chance at survival. Therefore, it is easy to understand the significant impact that development of grade 3-4 toxicities from immunotherapy can have regarding the prognosis of a patient with melanoma. According to the NCCN guidelines, patients who develop any grade of GBS toxicity from their immunotherapy should have it permanently discontinued [18].

Our case raises two important considerations: the first is whether it is reasonable to re-challenge a patient who has developed GBS as a result of prior treatment with a different class of immunotherapy. The second is whether the difference in the target for immune checkpoint inhibition between the different classes of immunotherapy can explain why our patient did not suffer a re-occurrence of her Guillain-Barré-like neuropathy.

Another important aspect highlighted by this case is the differences in the management of patients with idiopathic GBS and immunotherapy-associated GBS. With idiopathic GBS, a favorable response is typically seen after a single course of IVIG or plasma exchange, with no role of corticosteroids in this setting. The chance of recurrence after initial treatment is also low. However, treatment of immunotherapy-associated GBS requires the use of corticosteroids upfront to achieve a response and typically involves prolonged immunologic therapy due to a high chance of recurrence, presumably from the longstanding effects of immune checkpoint inhibition. By understanding these differences in management, our aim is to have oncologists actively engage with neurologists to impart this knowledge when treating patients who develop GBS from immunotherapy. 
Muralikrishnan et al.: Treatment Considerations for Pembrolizumab-Induced GBS

Toxicity in Melanoma

We hope that our case report not only adds to the growing literature of immunotherapyrelated side effects but also stimulates a discussion and further studies into whether a re-challenge with a different class of immunotherapy in the setting of GBS toxicity is a reasonable option for patients with metastatic, unresectable melanoma who have limited options.

\section{Conclusions}

Our case highlights an important clinical decision faced by oncologists when treating patients who develop Guillain-Barré type toxicity from immunotherapy. Although we generally agree with the NCCN guidelines for discontinuation of immunotherapy for any grade of GBS toxicity, we do feel that the decision to re-challenge with a different class of immunotherapy is reasonable in very specific circumstances, as seen in our particular case.

\section{Statement of Ethics}

Consent for publication was obtained from the patient.

\section{Disclosure Statement}

The authors declare that they have no competing interests.

\section{Funding Sources}

Lara K. Ronan: Hitchcock Foundation; Diamond Endowment Fund Dartmouth Hitchcock; National Endowment for the Arts; Reeves Foundation.

\section{Author Contributions}

Sivraj Muralikrishnan: writing and formulation of the manuscript.

Lara K. Ronan: patient care, authorship, editing.

Shodeinde Coker: patient care, manuscript revision.

Paula K. Rauschkolb: patient care, manuscript review.

Keisuke Shirai: patient care, authorship, editing.

\section{References}

1 Reck M, Rodríguez-Abreu D, Robinson AG, Hui R, Csoszi T, Fulop A, et al. Pembrolizumab versus chemotherapy for PD-L1-positive non-small-cell lung cancer. N Engl J Med. 2016 Nov;375(19):1823-33.

2 Long GV, Atkinson V, Ascierto PA, Robert C, Hassel JC, Rutkowski P, et al. Effect of nivolumab on health-related quality of life in patients with treatment-naïve advanced melanoma: results from the phase III CheckMate 066 study. Ann Oncol. 2016 Oct;27(10):1940-6.

3 Bellmunt J, de Wit R, Vaughn DJ, Fradet Y, Lee JL, Fong L, et al. Pembrolizumab as second-line therapy for advanced urothelial carcinoma. N Engl J Med. 2017;376(11):1015-26.

4 Wang DY, Salem J, Cohen JV, Chandra S, Menzer C, Ye F, et al. Fatal toxic effects associated with immune checkpoint inhibitors: a systematic review and meta-analysis. JAMA Oncol. 2018;4(12):1721-8.

5 Naidoo J, Page DB, Li BT, Connell LC, Schindler K, Lacouture ME, et al. Toxicities of the anti-PD-1 and anti-PDL1 immune checkpoint antibodies. Ann Oncol. 2015;26(12):2375-91. 
6 Wilgenhof S, Neyns B. Anti-CTLA-4 antibody-induced Guillain-Barre syndrome in a melanoma patient. Ann Oncol. 2011;22:991-3.

7 Gaudy-Marqueste C, Monestier S, Franques J, Cantais E, Richard MA, Grob JJ. A severe case of ipilimumabinduced Guillain-Barre syndrome revealed by an occlusive enteric neuropathy: a differential diagnosis for ipilimumab-induced colitis. J Immunother. 2013;36:77-8.

8 Fukumoto Y, Kuwahara M, Kawai S, Nakahama K, Kusunoki S. Acute demyelinating polyneuropathy induced by nivolumab. J Neurol Neurosurg Psychiatry. 2018 Apr;89(4):435-7.

9 Day D, Hansen AR. Immune-related adverse events associated with immune checkpoint inhibitors. BioDrugs. 2016;30:571-84.

10 Maur M, Tomasello C, Frassoldati A, Dieci MV, Barbieri E, Conte P. Posterior reversible encephalopathy syndrome during ipilimumab therapy for malignant melanoma. J Clin Oncol 2012;30:e76-8.

11 Bot I, Blank CU, Boogerd W, Brandsma D. Neurological immune-related adverse events of ipilimumab. Pract Neurol. 2013;13:278-80.

12 Rupareliya C, Naqvi S, Jani VB. Acute inflammatory demyelinating polyneuroradiculopathy with ipilimumab in metastatic melanoma: a case report and review of literature. Cureus. 2017;9(6):e1310.

13 Ryota T, Hiroshi M, Yasushi T, Kumi Y, Takumi H, Akiko I, et al. Nivolumab-induced chronic inflammatory demyelinating polyradiculoneuropathy mimicking rapid-onset Guillain-Barré syndrome: a case report. Jpn J Clin Oncol. 2016 Sep;46(9):875-8.

14 De Maleissye MF, Nicolas G, Saiag P. Pembrolizumab-induced demyelinating polyradiculopathy. N Engl J Med. 2016;375:296-7.

15 Manam R, Martin JL, Gross JA, Chaudhary D, Chowdhary S, Espinosa PS, et al. Case reports of pembrolizumabinduced acute inflammatory demyelinating polyneuropathy. Cureus. 2018 Sep;10(9):e3371.

16 Long GV, Schachter J, Ribas A, Arance AM, Grob JJ, Mortier L, et al. 4-year survival and outcomes after cessation of pembrolizumab (pembro) after 2-years in patients (pts) with ipilimumab (ipi)-naive advanced melanoma in KEYNOTE-006. J Clin Oncol. 2018;36(Suppl; abstr 9503).

17 Hodi FS, Chiarion-sileni V, Gonzalez R, Grob JJ, Rutkowski P, Cowey CL, et al. Nivolumab plus ipilimumab or nivolumab alone versus ipilimumab alone in advanced melanoma (CheckMate 067): 4-year outcomes of a multicentre, randomised, phase 3 trial. Lancet Oncol. 2018;19(11):1480-92.

18 National Comprehensive Cancer Network. Management of immunotherapy-related toxicities (version 1.2019) [accessed 2019 Jan 16]. Available from: https://www.nccn.org/professionals/physician_gls/pdf/immunotherapy.pdf. 